\title{
La vita, le lingue, les enfants et moi
}

\section{Luana Vergari}

\section{(2) OpenEdition}

\section{Journals}

\section{Edizione digitale}

URL: http://journals.openedition.org/esp/2490

DOI: $10.4000 /$ esp.2490

ISSN: 2532-0319

\section{Editore}

Centre d'Information sur l'Éducation Bilingue et Plurilingue

\section{Edizione cartacea}

Data di pubblicazione: 1 giugno 2018

Paginazione: 85-87

ISSN: 1127-266X

\section{Notizia bibliografica digitale}

Luana Vergari, «La vita, le lingue, les enfants et moi », Éducation et sociétés plurilingues [Online], 44

2018, Messo online il 08 février 2019, consultato il 14 septembre 2020. URL : http://

journals.openedition.org/esp/2490 


\section{LA VITA, LE LINGUE, LES ENFANTS ET MOI}

\section{Luana VERGARI}

Je m'appelle Luana Vergari. Fe suis italienne, j'habite en France, je parle italien (ma langue maternelle), français (la langue que j'ai apprise ici en France), anglais (la première langue étrangère que j’ai étudiée), espagnol (une langue qui me donne le sourire). Fe peux lire le cyrillique car avant de déménager j’avais commencé à prendre des cours de russe, l'alphabet grec car j'ai étudié le grec ancien à l'école, j'apprends l'hirigana car les enfants l'adorent et ils posent plein de questions pendant les ateliers d'éveil aux langues que j'anime. Fe suis un peu dyslexique et dysorthographique. Encore toute petite j'avais essayé de convaincre ma mère que j'étais capable de parler loules les langues du monde.

Mots-clés: parler avec tout le monde, découvrir le monde, langue du coeur, Dulala, groupe de jeux bilingue, diversité, imagination

My name is Luana Vergari. I'm Italian, I live in France, I speak Italian (my mother tongue), French (the language I learned here in France), English (the first foreign language I learned in school), Spanish (a language that makes me smile). I know how to read in Cyrillic because before moving house I had begun studying Russian, the Greek alphabet because I had ancient Greek in school, I'm learning hiragana because the children love it and ask a lot of questions during the Language Awareness classes I give. I'm a little bit dyslexic and dysorthographic (trouble spelling). When I was little, I tried to convince my mother I could speak all the languages in the world.

Key-words: speak with everyone, discover the world, language of the hearl, Dulala, bilingual playgroup, diversity, imagination

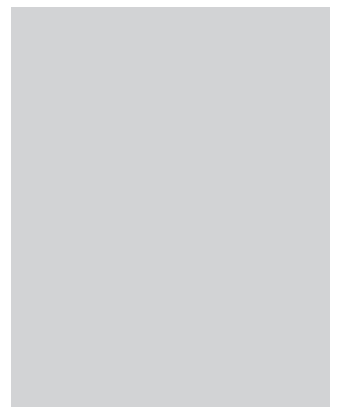

$V$

oilà ma biographie langagière! O forse no, dimentico la lingua che più di ogni altra ha avuto un peso nel mio immaginario, un posto speciale fra i miei affetti linguistici e che ha contribuito a creare e ad alimentare in me il desiderio di potermi esprimere in più lingue, parlare con chiunque, scoprire il mondo e le persone : il tedesco. Ich spreche kein Deutsch, la lingua della mia bisnonna Matilde e che sentivo a volte parlare da Nonna Lucia quando mi raccontava della sua bella infanzia e poi della giovinezza difficile divisa fra la guerra e la forza e la sfrontatezza di una mamma austriaca con due figlie che restate sole si dedicano al bar di famiglia. 
La vita, le lingue, les enfants et moi L. VergarI
Non ho mai sentito il bisogno d'imparare il tedesco. Forse perché il tedesco mi ha già fatto un regalo enorme: sapere che esistono delle lingue del cuore, quelle in cui puoi raccontare veramente te stesso. Il tedesco mi dato la consapevolezza che ogni lingua è in grado di raccontare un aspetto diverso di noi stessi. Ogni lingua è un nuovo racconto, una parte nuova di noi stessi che si apre al mondo, si svela, ci permette di nominare quello che prima un nome non lo aveva.

La mia passione per le lingue nasce in quei lunghi pomeriggi in cui ripetevo «Bitte» «Dank» e la buona notte che per mia nonna Lucia è sempre stata «Gute Nacht» fin quando sua mamma era lì per augurargliela.

Diversi anni dopo quei pomeriggi fatti di tee, tazze di porcellana e cucchiaini d'argento, ho avuto la fortuna d'incontrare a Parigi Dulala, un'associazione che si occupa di plurilinguismo. Ho seguito una prima formazione con loro e ho cominciato ad animare dei gruppi di gioco bilingue (francese/italiano) per bambini in età prescolare. Poi una seconda formazione «l'éveil aux langues» e sono arrivati les ateliers dans les écoles françaises. Poi è accaduto che per la prima volta nella vita ho sentito forte il bisogno e il desiderio di rimettere insieme tutte le mie competenze e così c̀ nato un album jeunesse «Les langues de Chat» editato da Dulala et qui a reçu le soutien du Ministère de la Culture ainsi que de la Ville de Montreuil et qui a remporté Le Label Européen de Langues. Da lì in poi la voglia di andare più lontano era inevitabile e ha portato alla creazione dell'Atelier Radiolem une association qui encourage l'apprentissage par le jeu, la création artistique et les nouvelles technologies. Un lieu d'innovation où toutes les langues sont les bienvenues et où on réalise des outils et des créations artistiques originales pour développer un projet pédagogique basé sur le respect et l'intégration.

La settimana scorsa animavo un atelier in una classe CP. I bambini erano divisi in tre squadre e dovevano indovinare la traduzione francese delle parole e le frasi che via via pronunciavo e scrivevo alla lavagna e che avevamo precedentemente scoperto insieme. A un certo punto scrivo in italiano la parola «Regalo» ed è il turno della squadra numero "Due" per rispondere. Martín ha subito alzato la mano sorridente, aveva lo sguardo sicuro di chi sa la risposta giusta. Quando gli ho dato la parola Martín mi ha detto «Regalo est la chose que tu donnes à quelqu'un le jour de son anniversaire, quelque chose qui est un secret caché dans un paquet». Ho risposto a Martín che era la risposta giusta e che però ora dovevamo capire insieme come si dice «Regalo» in francese. Ho chiesto a Martín se lo sapeva dire in spagnolo, la sua langue maternelle, mi ha detto 
La vita, le lingue, les enfants et moi

L. Vergari certo «Regalo», come in italiano. Allora l'ho sollecitato perché lo dicesse in francese, ma non lo ricordava. Gli ho detto «Cadeau» ma lui ha capito «Gâteau» e allora abbiamo convenuto che in fin dei conti il francese non è neanche la mia di langue maternelle e allora gli ho proposto di farci pronunciare bene le due parole dai suoi compagni di classe. Prima «Cadeau» e poi «Gâteau». Alla fine Martín ed io eravamo davvero soddisfatti e fieri di noi stessi. Tutti i bambini ci hanno detto che avevamo un'ottima pronuncia e che si sentiva bene la differenza fra «Torta» e «Regalo», «Gâteau» et «Cadeau». Se dovessi dire che senso abbia promuovere il bilinguismo e l'éveil aux langues nelle scuole, direi questo. Permettere a me e a Martín di sentirci accettati, permettere agli altri di vivere la nostra diversità come ricchezza per il gruppo... e poi diciamoci la verità, tutti vogliono avere in squadra Martín o Redeaun durante gli ateliers. Ł̀ facilissimo vincere quando hai dalla tua parte un bambino bilingue o trilingue perché può insegnarti tantissimo, addirittura la sua strategia per spostare tutte le competenze metalinguistiche da una lingua all'altra. 